\title{
Blau Syndrome, the prototypic auto-inflammatory granulomatous disease
}

Carine H Wouters ${ }^{1,2^{*}}$, Anne Maes ${ }^{2}$, Kevin P Foley ${ }^{4}$, John Bertin ${ }^{4}$ and Carlos D Rose ${ }^{3}$

\begin{abstract}
Blau syndrome is a monogenic disease resulting from mutations in the pattern recognition receptor NOD2, and is phenotypically characterized by the triad of granulomatous polyarthritis, dermatitis and uveitis. This paper reviews briefly the classical clinical features of the disease, as well as more recently described extra-triad symptoms. From an ongoing prospective multicenter study, we provide new data on the natural history of Blau syndrome, focusing on functional status and visual outcome. We also present an update of the range of different NOD2 mutations found in Blau syndrome as well as recent data on morphologic and immunohistochemical characteristics of the Blau granuloma. Finally, emerging insights into pathogenic mechanisms including activation of NOD2 signal transduction, and potential biomarkers of disease activity are discussed.
\end{abstract}

Keywords: Blau syndrome, Granulomatous diseases, NOD2, RIP2 kinase, Sarcoidosis, Uveitis

\section{Introduction and nomenclature}

In 1985, Blau syndrome (Blau) was described simultaneously by E Blau and D Jabs, as a familial granulomatous inflammatory disease with an autosomal dominant inheritance pattern $[1,2]$. Once the causative mutations in NOD2 were discovered [3] and later found to also be present in patients with Early Onset Sarcoidosis - a sporadic disease with the same phenotype [4,5] - , it became clear that Blau and Early Onset Sarcoidosis are the familial and sporadic forms respectively, of the same disease. The authors originally suggested a unifying name for these two conditions, namely Pediatric Granulomatous Arthritis but this term did not gain traction, partly because it concealed the systemic and visceral manifestations of the disease [6]. Further, arthritis although highly prevalent in this condition, is not a universal feature. In this paper we will utilize the term Blau syndrome to refer to both the sporadic and familial forms of the disease, recognizing that the presence or absence of a family history no longer has any relevance in terms of disease classification; of course the presence of an affected family member will be a valuable clinical feature for a diagnostician at the time of presentation.

\footnotetext{
*Correspondence: carine.wouters@uzleuven.be

'Department of Microbiology and Immunology, Pediatric Immunology, KU

Leuven - University of Leuven, B-3000 Leuven, Belgium

${ }^{2}$ Department of Pediatrics, Division Pediatric Rheumatology, University

Hospitals Leuven, B-3000 Leuven, Belgium

Full list of author information is available at the end of the article
}

Blau syndrome classically presents in early childhood as a triad of granulomatous dermatitis, arthritis and uveitis. Although there are exceptions, skin rash is the first symptom to appear, usually in the first year of life. Between the ages of $2-4$ years, a boggy polyarthritis is observed. Finally, uveitis develops in $60-80 \%$ of patients at around 48 months of age [6]. The majority of data presented in this review stem from two independent efforts. In 2005, the Blau syndrome International Registry (also referred to as the Pediatric Granulomatous Arthritis registry) was instituted. Thanks to the collaborative efforts of physicians taking care of Blau patients from around the world, genetic and clinical data based on retrospective review of medical records have been collected. This registry received support and is an integral part of the Eurofever project, since Blau syndrome is considered to be one of the monogenic auto-inflammatory diseases. Second, since 2009, a 5 -year prospective multicenter study to investigate the natural history of the disease and its functional impact, to promote biomarker discovery, and to gain a better understanding of relevant pathogenic pathways, is being conducted by the authors. Some of the preliminary findings from this latter international effort will be discussed herein.

One of the most fascinating aspects of Blau syndrome is the presence of non-caseating epithelioid and giant cell granulomas in affected tissues. In fact, Blau syndrome is 
the only auto-inflammatory disease associated with a gain of function mutation to show granulomatous inflammation. The morphologic characteristics of the "Blau granuloma" are similar to those found in sarcoidosis, which is not associated with NOD2 mutations. Detailed morphological and immunohistochemistry studies on biopsy tissue from Blau patients have provided some insights as to the relevant pathogenic pathways involved in the disease process [7]; however there is still a significant knowledge gap concerning a link between the canonical NOD2 signaling pathway and granuloma formation.

\section{Review}

\section{Classical clinical features}

Blau arthritis manifests as a polyarticular "boggy" synovitis and tenosynovitis which constitutes a characteristic phenotypical feature. Data from the Blau international registry revealed polyarticular arthritis at presentation in $96 \%$ of patients with a "boggy"/exuberant aspect in $75 \%$ [6]. Arthritis typically affects peripheral joints mainly wrists, knees, ankles, and proximal interphalangeal (PIP) joints of the hands. Other peripheral joints are less commonly affected and involvement of the axial skeleton and the temporo-mandibular joint has not been described. Tenosynovitis is equally a characteristic feature; tendon sheaths appear enlarged on examination; most often the extensor tendons of the wrist, the pes anserinus, peroneal and flexor tibialis tendon sheaths are affected. Despite the chronicity of exuberant arthritis, joint destruction is not commonly reported, and range of motion is relatively well preserved, especially in the larger joints. Conversely, the PIP joints often develop a contracture relatively early in the course of the disease and out of proportion to the degree of synovitis. This deformity, originally described as camptodactyly, may be the result of dysplastic changes, distinct from the inflammation-associated joint contracture as seen in polyarticular juvenile idiopathic arthritis (JIA) [8]. Figures 1 and 2 show the characteristic appearance of Blau arthritis. More detailed descriptions of the articular phenotype are available in other publications [9-14].

The rash is usually the first to appear in Blau; however, by the time the patients present to the rheumatologist all one may find is either a residual pigmented, mildly scaly rash on examination or simply a history of a "strange" rash during infancy. Clinically the most frequent appearance is that of an erythematous maculo-micropapular fine scaly rash on the trunk and extremities in an infant. Intense erythematous rashes have been described [15]; more commonly, the rash is misdiagnosed as atopic dermatitis, or when desquamation is intense, as ichthyosis vulgaris. Blau syndrome also has to be considered in the differential diagnosis of neonatal dermatitis. In one report, a generalized erythematous micropapular dermatitis was described in an infant of 1 week old at presentation with a skin

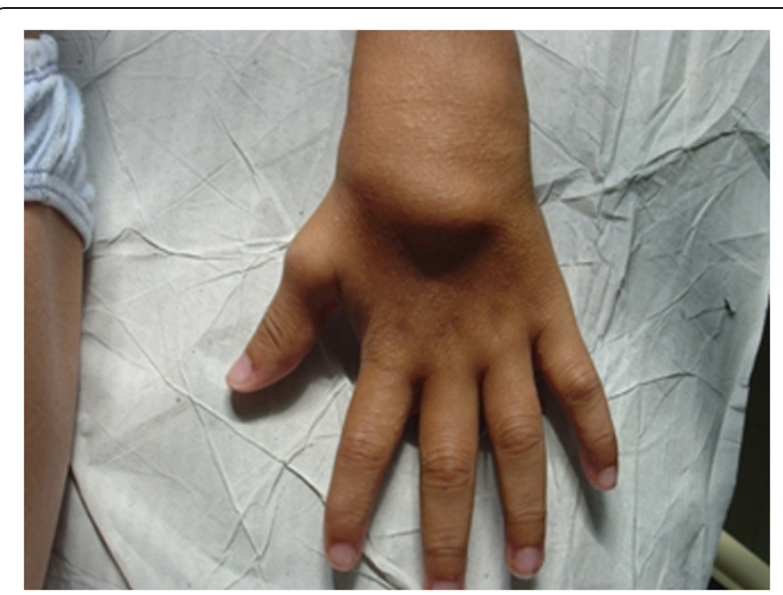

Figure 1 Exuberant 'boggy' synovitis of wrist in a child with Blau syndrome.

biopsy showing non-caseating granulomas [15]. Later the exanthema becomes tan-colored and may present a "dirty' scaly appearance (Figure 3). The later stage of the rash tends to last longer and may constitute a valuable diagnostic clue. When a skin biopsy is performed a non-caseating granulomatous inflammatory infiltrate located in the dermis is characteristic (Figure 4). In the Blau international registry, the skin has offered better diagnostic yield when compared to synovium, with confirmatory histology in more than $90 \%$ of the patients eventually diagnosed with Blau [6].

Uveitis presents as an insidious granulomatous iridocyclitis with posterior uveitis in many patients, and a potential evolution into a severe panuveitis with multifocal choroiditis. It is usually bilateral and associated with an increased risk of visual morbidity. About $80 \%$ of patients in both the retrospective international Blau registry and the prospective Blau cohort study developed ocular disease with a median age at onset of 4.4 years (unpublished data). Unlike the uveitis of JIA, which is

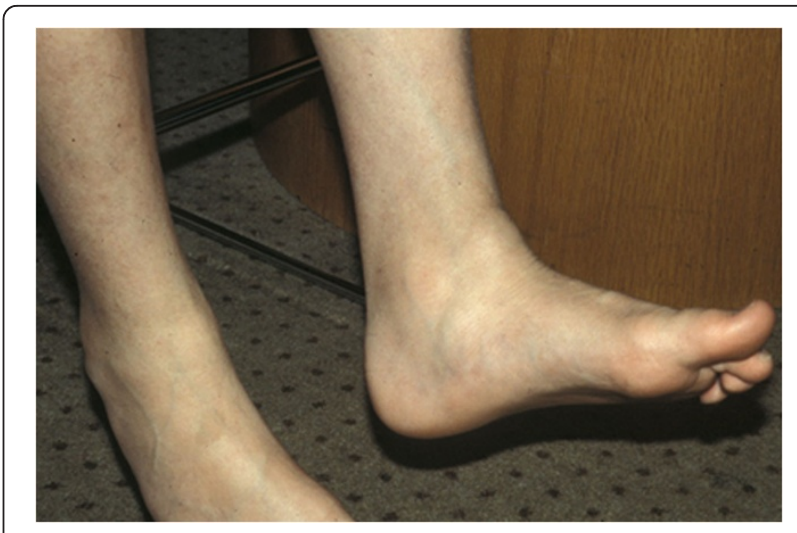

Figure 2 Tenosynovial swelling affecting tibialis posterior and peroneal tendons in Blau syndrome. 


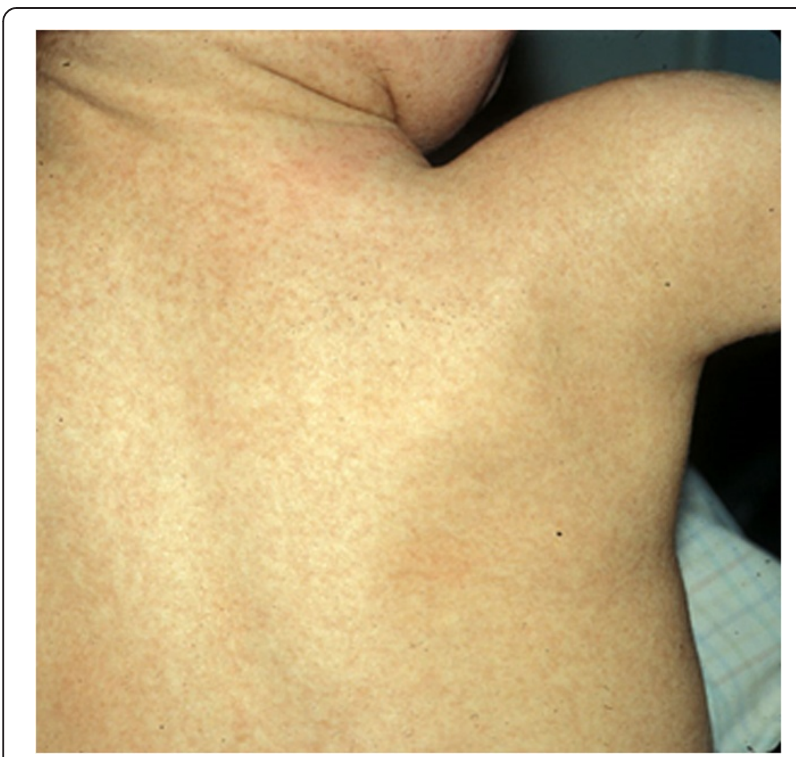

Figure 3 Tan colored "dirty" mildly scaly (late) rash.

almost always confined to the anterior uvea, involvement of the entire uveal tract with panuveitis was found in 75\% of Blau patients in the prospective cohort study (unpublished data). Granulomatous uveitis may be suspected on slit lamp examination by the presence of nodular peripheral keratic precipitates noted on the limbus [16]. The synechiae tend to be focal, and there can be nodules in the iris. Vitreous inflammation is common, and may remain persistently active for years. Posterior involvement is described as multifocal choroiditis.

\section{Expanded manifestations}

The first systematic study to show the importance of extratriad manifestations was published by Arostegui in 2007.

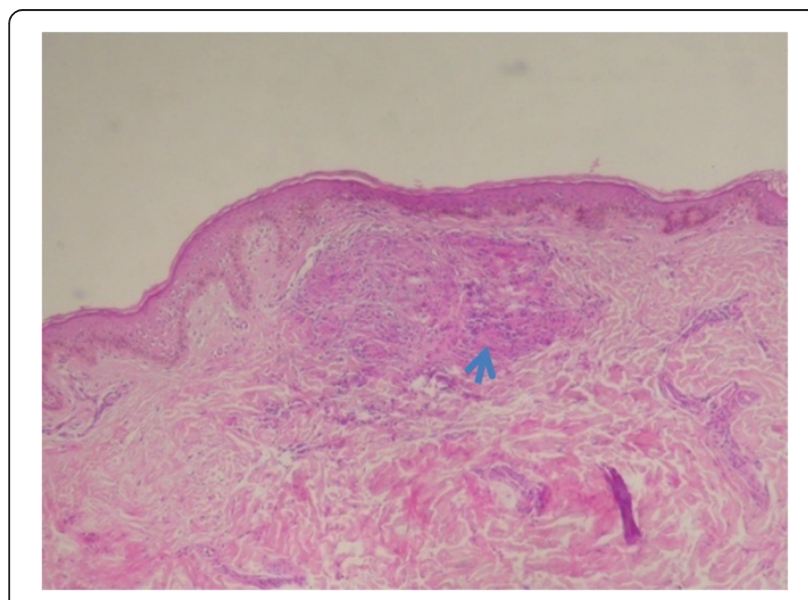

Figure $\mathbf{4}$ Granulomatous dermatitis. Inflammatory infiltrate of the subpapilar dermis with typical multinucleated giant cells within a non-caseating epithelioid cell Blau granuloma.
Most notably fever and erythema nodosum were detected quite commonly in this Spanish cohort [9]. As more patients were recruited in the Blau international registry we noticed expanded manifestations in about one third of the patients [17]. Features included fever, sialadenitis, lymphadenopathy, erythema nodosum, leukocytoclastic vasculitis, transient neuropathies, granulomatous glomerular and interstitial nephritis, interstitial lung disease, arterial hypertension, pericarditis, pulmonary embolism, hepatic granulomas, splenic involvement and chronic renal failure.

Some of the extra-triad manifestations are of particular importance to be aware of when following Blau patients. The association between large vessel vasculitis and Blau syndrome was reported before the genetic mutation was discovered [18-21]. Other investigators reported silent aortitis in a patient with Blau syndrome [22]. We recently described a case of Takayasu's-like arteritis in a child from India with a novel NOD2 mutation [23].

Also of note, two young patients from the international registry developed severe systemic hypertension; although digital angiography could not demonstrate renovascular disease, the response to ACE inhibitors was manifest [17]. A young patient with a typical triad phenotype without visceral manifestations for most of his life succumbed to acute onset intractable pulmonary hypertension at the age of 22 years, within a few months from the first symptoms of mild dyspnea (unpublished observation). Hence pulmonary and systemic hypertension may be seen in the course of Blau syndrome, requiring careful vigilance.

Cranial neuropathies, particularly facial palsy have also been documented in Blau [2,21], but central nervous system involvement has not been reported. A patient with Blau syndrome and a new onset of a seizure disorder was recently published; yet the relationship with the disease process was not clearly demonstrated [24]. Typical central nervous system manifestations seen in adult sarcoidosis, namely meningeal and white matter disease have not been described in Blau.

Interstitial lung disease, a major feature of adult sarcoidosis, was originally not considered part of the Blau phenotype. The first case with a documented NOD2 mutation was reported by our group [25]. Pneumonitis in this child was characterized by ground glass infiltrates in the upper lobes easily controllable with a few weeks of corticosteroids, without sequelae and no recurrence while on anti-TNF therapy. Interstitial lung disease was observed in three additional patients of the prospective Blau study.

Erythema nodosum has been mentioned frequently; in fact it is the second most common cutaneous manifestation of Blau syndrome. Also leukocytoclastic vasculitis has been documented in a patient with Blau [26]. 
Renal involvement has been described as an interstitial nephritis in the context of Blau syndrome before the mutation was known [27]. The first case of acute glomerular and interstitial nephritis with histological documentation of granulomatous inflammation both in the interstitium and the glomeruli was reported more recently [26].

Finally, involvement of the reticuloendotelial system is not uncommon. We found generalized lymphadenopathies and splenomegaly in more than one-fifth of patients in the Blau prospective study. Hepatomegaly can be seen, but liver enzymes and liver function are mostly normal. We have reported hepatic granulomas on a liver biopsy in a child with Blau syndrome without evidence of hepatic injury neither histologically nor by laboratory evaluation [28].

\section{Disease course}

To date all studies on Blau have been either short case series or multicenter registries [6,9] based on retrospectively collected data. The natural history of Blau and its functional consequences remain largely unknown. An ongoing prospective international study aiming at collecting data on disease course, and evolution of articular, ocular and visceral disease was established in 2009. At the time of writing this review, baseline visit data on the first 31 recruited Blau patients with a median disease course of 12.8 yrs were available (manuscript under review).
At the time of evaluation, more than fifty percent of Blau patients had articular deformities, with camptodactyly (flexion contracture at PIP joints) being most commonly observed. Radiographs of hands and wrist very rarely showed erosions, however some morphological changes of radial, ulnar and carpal bones were apparent [8]. Functional capacity, assessed by CHAQ and HAQ, showed normal function in more than one-third of patients, mild impairment in one-third and moderate/severe in almost one-third. Almost half of Blau patients reported a moderate to severe impact of the disease on their global well-being, and moderate to severe pain [8].

The large majority of patients with uveitis had active eye involvement after 15 yrs. More than one-third had ocular complications (including band keratopathy, increased intraocular pressure, cataracts, optic atrophy, macular edema and retinal detachment), and almost two-thirds had visual loss [8]. Figure 5 depicts the distribution of visual outcome at baseline.

At present, there is no evidence for an effective treatment for all patients with Blau. In the prospective Blau cohort study, more than two-thirds of Blau patients received medical therapy, often combining systemic steroids, immunosuppressive and/or biologic drugs to control both uveitis and arthritis. TNF-antagonists are the most commonly used biological therapy and seemingly useful in achieving partial control of articular disease. A few investigators however have reported a dramatic response

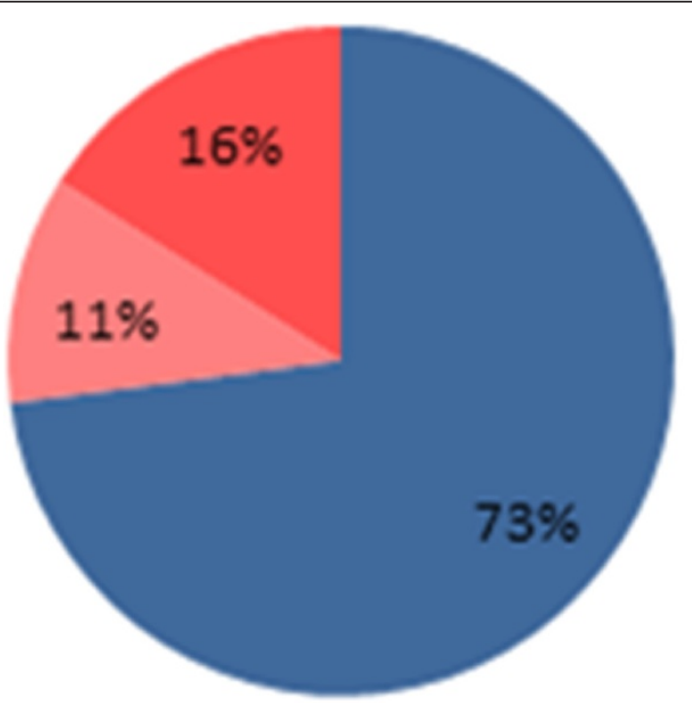

n Normal vision

noderate visual impairment (LogMAR>0,5)

alind ( LogMAR $>1,0)$

Figure 5 Distribution of visual acuity in a cohort of 22 patients with Blau syndrome associated uveitis after an average follow up of 12.5 years. Visual acuity was converted into logMAR units by calculating the -Log of the visual acuity in decimal Snellen scale in meters. 
both clinically and biologically to IL-1 inhibition $[9,29]$. The observation of persistently active disease in a majority of Blau patients in this study underlines the need for development of effective targeted therapies.

\section{The NOD2 genotypic spectrum}

In the initial report in which NOD2 was identified as the causative gene in Blau syndrome, three different single base pair mutations were described in 4 families [3]. Two of these families exhibited the same mutation, encoding an amino acid substitution of arginine to tryptophan at position 334 (R334Q), one family had an R334W, and one other a L469F substitution. The following year, Wang et al. published their study on the genetic analysis of NOD2 coding regions based on 10 families with Blau syndrome including members of the original family published by E. Blau [21]. This paper mentioned a surprisingly low frequency of NOD2 mutations in patients with Blau; 5 of the 10 families did not show any mutations, yet the description of the phenotypes was not very detailed. A large series reported by investigators from Japan identified NOD2 mutations in $90 \%$ of their cases [30]. In the international Blau registry, all patients with the classical clinical triad phenotype had a NOD2 mutation [6].

Over time the number of different NOD2 mutations associated with Blau has expanded greatly [31-36]. An update was published in 2011 by the authors [37], and new Blau-associated NOD2 mutations are being cataloged regularly in the Infevers registry [38].

Most of the Blau mutations reported to date are at or near the nucleotide-binding NOD/NACHT domain of the NOD2 protein, yet they can also be found extending into the C-terminal region characterized by a leucine-rich repeat (LRR) structure. Figure 6 depicts the distribution of NOD2 mutations found among 82 patients enrolled in the international registry confirming that the majority of patients have either R334Q or R334W mutations, and most of them are within/near the NACHT domain. We found one individual with a classical phenotype and a substitution at position 802, corresponding to the LRR region (Figure 6). An E600A mutation was also found in affected members of a three-generation family with isolated granulomatous uveitis, and also reported in an individual with classical Blau [17].

It is unclear whether the type and/or location of the NOD2 mutation has an effect on the clinical features. In a study from Japan, an increased frequency and severity of ocular involvement was reported in association with R334W substitution [39], a finding we could not confirm in neither the international Blau registry nor the current prospective study.

Interestingly, although the penetrance of Blau-associated NOD2 mutations is very high, we reported a family with an E383K substitution, in which 2 members had the typical Blau phenotype and 4 additional members (including one adult) with the same substitutions, were asymptomatic [40].

Incomplete phenotypes are not uncommon; about $15-20 \%$ of patients with Blau syndrome do not develop eye disease. In addition, we recently described a young girl with a novel G464W substitution, who presented with an atypical phenotype comprising mild oligoarthritis, a Takayasu's like large-vessel arteritis, but absence of rash and no uveitis [23].

\section{Blau granuloma morphology and immunohistochemistry}

Granulomas are organized inflammatory infiltrates characterized by a core of macrophages, epithelioid cells, multinucleated giant cells and a corona of lymphocytes and a variable presence of fibroblasts. Blau is the only auto-inflammatory disease with a gain-of-function mutation and granulomatous inflammation in affected tissues and organs. Interestingly, granulomas also commonly occur in the gastrointestinal tract in Crohn's disease, and a subset of Crohn's patients carry polymorphisms in NOD2, which are generally believed to confer loss of function [41].

\section{NOD2 mutations in Blau}

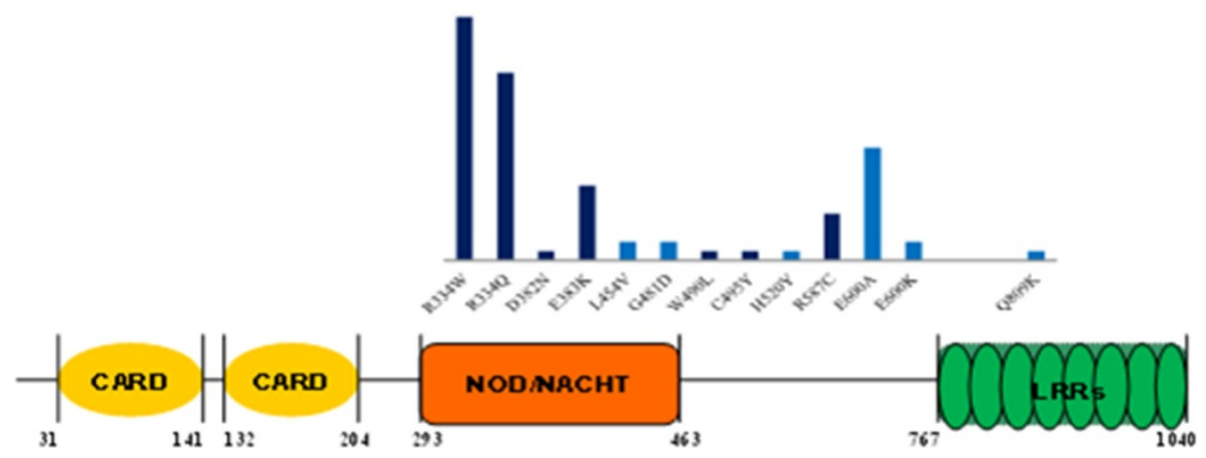

Figure 6 Distribution of NOD 2 mutations in 82 patients of the International Blau registry (6). 
We have performed an in-depth morphological and immunohistochemical study on granuloma-containing biopsies from Blau patients as well as from patients with Crohn's disease and NOD2 polymorphisms. Our data show that Blau granulomas display a distinct morphology characterized by large polycyclic granulomas with dense lymphocytic coronas, reflecting an exuberant inflammatory response, and in line with a gain-of-function mutation in NOD2. In contrast, biopsies from Crohn's patients show isolated granulomas with loose lymphocytic borders and sclerosis of surrounding tissue, which are also seen in immunodeficiency disorders, and therefore compatible with loss-of-function variants of NOD2 [7].

In these studies, using immunohistochemistry, an abundant inflammatory cytokine expression was observed in situ in Blau granulomas. A prominent expression of IFN- $\gamma$ was seen, which is in accordance with an important role for Th1 lymphocytes in granulomatous inflammation, as reported earlier in adult sarcoidosis [42]. In addition, we found a very high expression of IL-6, TGF- $\beta$ and IL-17 as well as an increased expression of the IL-23 receptor on granuloma cells (Figure 7) [7], compatible with activation of the Th17 lymphocyte axis. A role for NOD2 in the induction of the Th17 axis has been reported by Van Beelen et al. who showed that in vitro stimulation of human dendritic cells with the NOD2-ligand muramyl dipeptide resulted in promotion of IL-17 expression and Th17 differentiation from memory T cells. Conversely, NOD2-defective dendritic cells from Crohn's disease patients had marked impairment in inducing Th17 polarization from memory
T-cells [43]. Activation of Th-17 cells in Blau syndrome granulomas would be compatible with a gain-of-function mutations of NOD2 causing the disease. Of interest, a role for both Th1 and Th17 cells in adult sarcoidosis has been reported recently [42].

In Blau granulomas, we also have documented widespread extensive emperipolesis (cell-in-cell phenomenon) of lymphocytes within multinucleated giant cells, which is associated with multinucleated giant cell death, a finding of interest in view of the recently reported role of NOD2 in autophagy (Figure 7) [7].

\section{Blau syndrome new insights into pathogenesis}

A full account of the biology of the NOD2 protein is beyond the scope of this article, and has been extensively reviewed elsewhere $[44,45]$. Briefly, NOD2 is a member of the NOD-like receptor (NLR) family of pattern recognition receptors, which are involved in inflammation and innate immune defense against invading pathogens. NOD2 has a tripartite structure with two N-terminal CARD domains, one centrally located NOD/NACHT domain and a $\mathrm{C}$-terminal domain comprised of ten leucine-rich repeat (LRR) motifs (Figure 6). The LRR domain binds the NOD2 ligand muramyl dipeptide (MDP), a degradation product of ubiquitous bacterial cell wall peptidoglycan (Figure 6).

The classical pathway of NOD2 activation begins with oligomerization of NOD2 after ligand-receptor engagement (Figure 8). It has been postulated that in its unstimulated state, NOD2 is autoinhibited via intramolecular interaction between its LRR and CARD domains. Engagement of MDP

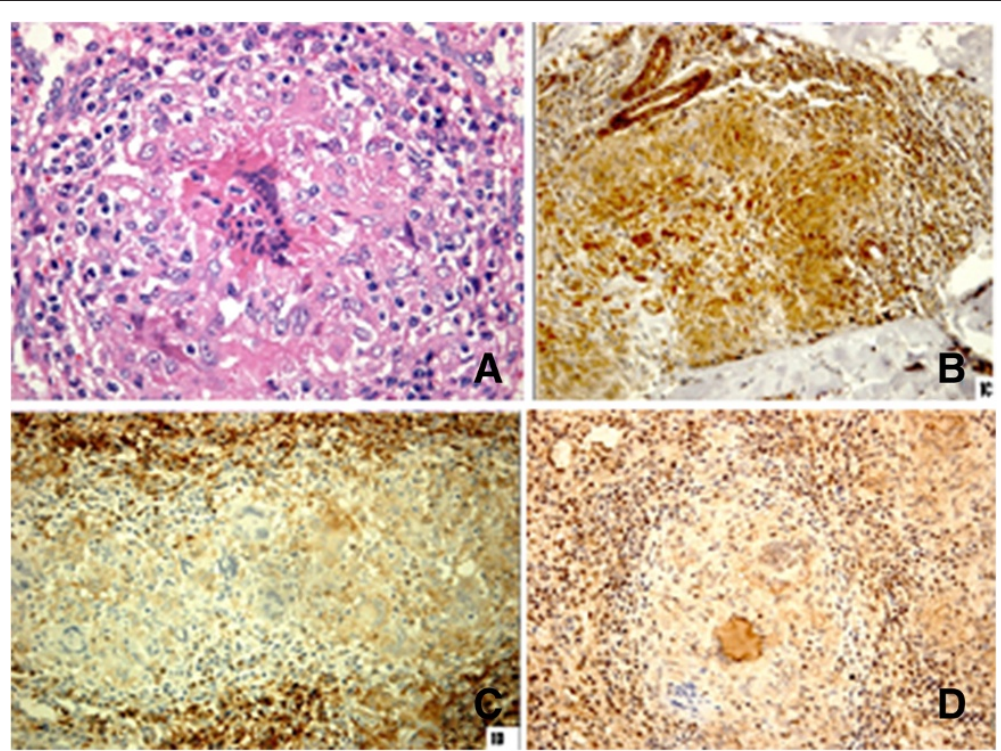

Figure 7 Morphological and immunohistochemical characteristics of Blau granulomas. Brown colour denotes positive cytokine staining. A (top left). H \& E staining showing prominent lymphocyte corona, emperipoletic lymphocytes, and multinucleated giant cell death with fragmented cytoplasm and pycnotic nuclei. Using immunohistochmistry dense staining was observed for IFN- $\gamma$ (B, top right, low magnification showing several granulomas), IL-6 (C, bottom left, single granuloma showing predominant staining in corona) and IL-17 (D, bottom right, single granuloma with giant cell in center showing strong $\|-17$ staining). 


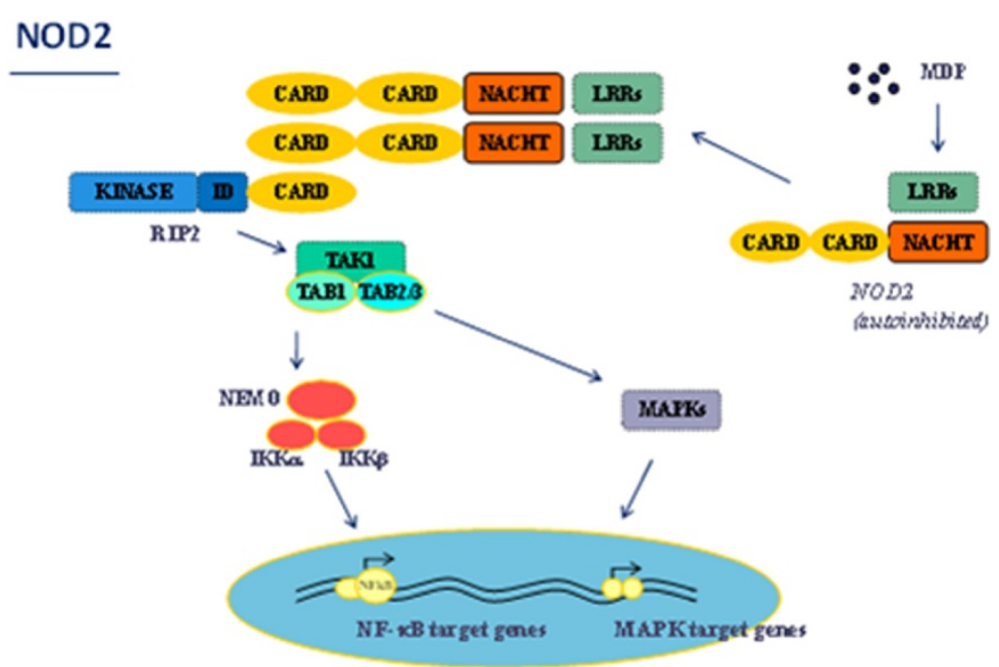

Figure 8 Schematic representation of the NOD2 signaling pathway. Upon binding of its natural ligand (MDP), the NOD2 protein unfolds and oligomerizes through the NACT domain. This is followed by recruitment of RIP2 kinase protein through CARD-CARD interactions and activation of downstream transcriptional factors (NF-KB and MAP kinase) and secretion of inflammatory cytokines.

ligand leads to unfolding from this autoinhibited state and oligomerization via the exposed NOD/NACHT domain. This results in activation and further engagement of RIP2 kinase, its immediate downstream signaling partner via interaction between the CARD domains of each protein. Consequent activation of the key downstream signaling molecules NF- $\mathrm{kB}$ and MAP kinase ultimately results in production of inflammatory cytokines such as IL-1 $\beta, \mathrm{IL}-6$, IL-8, TNF- $\alpha$, and a variety of other cytokines, chemokines and adhesion molecules. The physiological role of NOD2 has been expanding beyond being an innate line of defense against intracellular bacterial infections and equally involves a role in the defense against Toxoplasma [46], as a viral pattern recognition receptor [47], and in the induction of the autophagy process initiated by intracellular bacteria eg Shigella flexneri [48].

The hypothesis that NOD2 mutations in Blau syndrome act in a gain of function manner was initially suggested by the autosomal dominant inheritance pattern of the disease [3]. Subsequently, studies by two groups demonstrated that over-expression of NOD2 carrying Blau mutations in transfected cells resulted in the hyper-activation of an $\mathrm{NF}-\kappa \mathrm{B}$ responsive reporter gene, whereas expression of NOD2 carrying loss of function Crohn's disease mutations failed to activate the same reporter [30,49]. These results are consistent with the observation that Blau NOD2 mutations occur in amino acid positions homologous to known gain of function mutations occurring in the related pattern recognitions receptor NLRP3 which lead to spontaneous IL- $1 \beta$ production in the autoinflammatory Cryopyrin-associated periodic syndromes (CAPS) [50].
Based on these studies, NF- $\mathrm{kB}$ activation and excess inflammatory cytokine production as a consequence of dominant gain of function mutations in NOD2 has become a widely accept concept in the field. However, we and other groups have observed that, in contrast to NLRP3-mutated CAPS, peripheral blood cells from Blau patients do not spontaneously produces inflammatory cytokines, and are in fact resistant to stimulation by the NOD2 ligand MDP $[51,52]$. Although this may appear to be at odds with a gain of function model, it should be remembered that these peripheral cell populations may not reflect the activation state of immune cells present within granulomas in diseased tissue, where, as described above, we have seen evidence of constitutive inflammation. Indeed, we have also detected increased expression of S100A12 in Blau patient plasma, which is a marker of neutrophil-mediated inflammation [52]. Reconciling all these observations will obviously require further work, particularly to understand the relationship between Blau gain of function mutations and the mechanism of granuloma formation.

\section{Conclusion}

The clinical spectrum of NOD2-associated granulomatous inflammatory diseases continues to expand. NOD2 mutations associated with Blau syndrome extend beyond the NACHT domain. The rarity of Blau syndrome has made it challenging to fully characterize the disease. However, the recent creation of an international registry and the ongoing natural history study are providing novel insights into the symptomology and etiology of this fascinating genetic disorder. 
With more data available on the natural history of Blau syndrome, the need for more effective therapies in a substantial subset of patients is clear. Increased knowledge on NOD2 signaling and function remains essential to advance our understanding of NOD2-hyperactivation diseases.

\section{Competing interests}

Dr Wouters was awarded an unrestricted grant from GlaxoSmithKline to study Blau syndrome. Dr Rose is a Co-investigator on that grant.

\section{Authors' contributions}

CHW and CDR were involved in conceptualization, design, analysis, interpretation, provision of clinical expertise, and manuscript preparation. AM was involved in screening citations and full text and manuscript preparation. KF and JB were involved in manuscript editing, and provided scientific expertise. All authors read and approved the final manuscript.

\section{Acknowledgements}

We remain grateful to Tammy Martin PhD who was responsible for the initial genotyping work and her insights in disease pathogenesis have stimulated the author's interest in the disease.

Currently the Blau repository is under the direction of Juan J Arostegui MD to whom the authors remain indebted for his work on genotyping and interpreting genetic data from participants and patients from around the world.

\section{Author details}

${ }^{1}$ Department of Microbiology and Immunology, Pediatric Immunology, KU Leuven - University of Leuven, B-3000 Leuven, Belgium. '2Department of Pediatrics, Division Pediatric Rheumatology, University Hospitals Leuven, B-3000 Leuven, Belgium. ${ }^{3}$ Division of Rheumatology Alfred I. duPont Hospital for Children, Pediatrics Thomas Jefferson University, Wilmington, Delaware, USA. ${ }^{4}$ Pattern Recognition Receptor Discovery Performance Unit, Immuno-inflammation Therapeutic Area, GlaxoSmithKline, Collegeville, Pennsylvania, USA.

\section{Received: 9 May 2014 Accepted: 27 July 2014}

Published: 6 August 2014

\section{References}

1. Blau EB: Familial granulomatous arthritis, iritis, and rash. J Pediatr 1985, 107:689-693.

2. Jabs DA, Houk JL, Bias WB, Arnett FC: Familial granulomatous synovitis, uveitis, and cranial neuropathies. Am J Med 1985, 78:801-804.

3. Miceli-Richard C, Lesage S, Rybojad M, Prieur AM, Manouvrier-Hanu S, Hafner R, Chamaillard M, Zouali H, Thomas G, Hugot JP: CARD15 mutations in Blau syndrome. Nat Genet 2001, 29:19-20.

4. Kanazawa N, Matsushima S, Kambe N, Tachibana T, Nagai S, Miyachi Y: Presence of a sporadic case of systemic granulomatosis syndrome with a CARD15 mutation. J Invest Dermatol 2004, 122:851-852.

5. Rose CD, Doyle TM, Mcllvain-Simpson G, Coffman JE, Rosenbaum JT, Davey MP, Martin TM: Blau syndrome mutation of CARD15/NOD2 in sporadic early onset granulomatous arthritis. J Rheumatol 2005, 32:373-375.

6. Rose CD, Wouters CH, Meiorin S, Doyle TM, Davey MP, Rosenbaum JT, Martin TM: Pediatric granulomatous arthritis: an international registry. Arthritis Rheum 2006, 54:3337-3344.

7. Janssen CE, Rose CD, De Hertogh G, Martin TM, Bader Meunier B, Cimaz R, Harjacek M, Quartier P, Ten Cate R, Thomee C, Desmet VJ, Fischer A, Roskams T, Wouters CH: Morphologic and immunohistochemical characterization of granulomas in the nucleotide oligomerization domain 2-related disorders Blau syndrome and Crohn disease. J Allergy Clin Immunol 2012, 129:1076-1084.

8. Rose CD, Cimaz R, Thomee C, Khubchandani RP, Espada G, Russo R, Harjacek M, Bader Meunier B, Brissaud P, Wulffraat N, Vastert S, Merino R, Naranjo-Hernandez A, Oliveira-Knupp S, Mackensen F, Arostegui J, Anton J, Fernandez-Martin J, Wouters C: Blau Prospective Cohort Study: Articular and Ocular Outcomes. Lausanne, Switzerland: 17th Congress of ISSAID, International Society of Systemic Auto-Inflammatory Diseases; 2013.
9. Arostegui Jl, Arnal C, Merino R, Modesto C, Antonia Carballo M, Moreno P, Garcia-Consuegra J, Naranjo A, Ramos E, de Paz P, Ruis J, Plaza S, Yagüe J: NOD2 gene-associated pediatric granulomatous arthritis: clinical diversity, novel and recurrent mutations, and evidence of clinical improvement with interleukin-1 blockade in a Spanish cohort. Arthritis Rheum 2007, 56:3805-3813.

10. Hafner R, Vogel P: Sarcoidosis of early onset. A challenge for the pediatric rheumatologist. Clin Exp Rheumatol 1993, 11:685-691.

11. North AF Jr, Fink CW, Gibson WM, Levinson JE, Schuchter SL, Howard WK, Johnson NH, Harris C: Sarcoid arthritis in children. Am J Med 1970, 48:449-455.

12. Pastores GM, Michels W, Stickler GB, Su WP, Nelson AM, Bovenmyer DA: Autosomal dominant granulomatous arthritis, uveitis, skin rash, and synovial cysts. J Pediatr 1990, 117:403-408.

13. Raphael SA, Blau EB, Zhang WH, Hsu SH: Analysis of a large kindred with Blau syndrome for HLA, autoimmunity, and sarcoidosis. Am J Dis Child 1993, 147:842-848.

14. Rose CD, Martin TM: Caspase recruitment domain 15 mutations and rheumatic diseases. Curr Opin Rheumatol 2005, 17:579-585.

15. Stoevesandt J, Morbach H, Martin TM, Zierhut M, Girschick H, Hamm H: Sporadic Blau syndrome with onset of widespread granulomatous dermatitis in the newborn period. Pediatr Dermatol 2010, 27:69-73.

16. Lindsley $C B$, Godfrey WA: Childhood sarcoidosis manifesting as juvenile rheumatoid arthritis. Pediatrics 1985, 76:765-768.

17. Rose CD, Arostegui JI, Martin TM, Espada G, Scalzi L, Yague J, Rosenbaum JT, Modesto C, Cristina Arnal M, Merino R, García-Consuegra J, Carballo Silva MA, Wouters CH: NOD2-associated pediatric granulomatous arthritis, an expanding phenotype: study of an international registry and a national cohort in Spain. Arthritis Rheum 2009, 60:1797-1803.

18. Gross KR, Malleson PN, Culham G, Lirenman DS, McCormick AQ, Petty RE: Vasculopathy with renal artery stenosis in a child with sarcoidosis. J Pediatr 1986, 108:724-726.

19. Rose CD, Eichenfield AH, Goldsmith DP, Athreya BH: Early onset sarcoidosis with aortitis-“juvenile systemic granulomatosis?". J Rheumatol 1990, 17:102-106.

20. Rotenstein D, Gibbas DL, Majmudar B, Chastain EA: Familial granulomatous arteritis with polyarthritis of juvenile onset. N Engl J Med 1982, 306:86-90.

21. Wang X, Kuivaniemi H, Bonavita G, Mutkus L, Mau U, Blau E, Inohara N, Nunez G, Tromp G, Williams CJ: CARD15 mutations in familial granulomatosis syndromes: a study of the original Blau syndrome kindred and other families with large-vessel arteritis and cranial neuropathy. Arthritis Rheum 2002, 46:3041-3045.

22. Mourad F, Tang A: Sinus of valsalva aneurysm in Blau's syndrome. J Cardiothorac Surg 2010, 5:16.

23. Khubchandani RP, Hasija R, Touitou I, Khemani C, Wouters CH, Rose CD: Blau arteritis resembling Takayasu disease with a novel NOD2 mutation. J Rheumatol 2012, 39:1888-1892.

24. Nia AE, Nabavi M, Nasab MM: Central nervous system involvement in Blau syndrome: a new feature of the syndrome? J Rheumatol 2007, 34:2504-2505

25. Becker ML, Martin TM, Doyle TM, Rose CD: Interstitial pneumonitis in Blau syndrome with documented mutation in CARD15. Arthritis Rheum 2007, 56:1292-1294

26. Meiorin SM, Espada G, Costa CE, Tartara A, De Matteo E, Wouters C, Martin TM, Rose CD: Granulomatous nephritis associated with R334Q mutation in NOD2. J Rheumatol 2007, 34:1945-1947.

27. Ting SS, Ziegler J, Fischer E: Familial granulomatous arthritis (Blau syndrome) with granulomatous renal lesions. J Pediatr 1998, 133:450-452.

28. Saini SK, Rose CD: Liver involvement in familial granulomatous arthritis (Blau syndrome). J Rheumatol 1996, 23:396-399.

29. Simonini G, Xu Z, Caputo R, De Libero C, Pagnini I, Pascual V, Cimaz R: Clinical and transcriptional response to the long-acting interleukin-1 blocker canakinumab in Blau syndrome-related uveitis. Arthritis Rheum 2013, 65:513-518.

30. Kanazawa N, Okafuji I, Kambe N, Nishikomori R, Nakata-Hizume M, Nagai S, Fuji A, Yuasa T, Manki A, Sakurai Y, Nakajima M, Kobayashi H, Fujiwara I, Tsutsumi H, Utani A, Nishigori C, Heike T, Nakahata T, Miyachi Y: Early-onset sarcoidosis and CARD15 mutations with constitutive nuclear factor-kappaB activation: common genetic etiology with Blau syndrome. Blood 2005, 105:1195-1197. 
31. Milman N, Ursin K, Rodevand E, Nielsen FC, Hansen TV: A novel mutation in the NOD2 gene associated with Blau syndrome: a Norwegian family with four affected members. Scand J Rheumato/ 2009, 38:190-197.

32. Okada S, Konishi N, Tsumura M, Shirao K, Yasunaga S, Sakai H, Nishikomori $R$, Takihara Y, Kobayashi M: Cardiac infiltration in early-onset sarcoidosis associated with a novel heterozygous mutation, G481D, in CARD15. Rheumatology 2009, 48:706-707.

33. Priori R, Bombardieri M, Spinelli FR, Merlin F, Miceli-Richard C, La Cava M Scavalli AS, Guerrisi R, Hugot JP, Valesini G: Sporadic Blau syndrome with a double CARD15 mutation. Report of a case with lifelong follow-up. Sarcoidosis Vasc Diffuse Lung Dis 2004, 21:228-231.

34. Sakai H, Ito S, Nishikomori R, Takaoka Y, Kawai T, Saito M, Okafuji I, Yasumi T, Heike T, Nakahata T: A case of early-onset sarcoidosis with a six-base deletion in the NOD2 gene. Rheumatology 2010, 49:194-196.

35. van Duist MM, Albrecht M, Podswiadek M, Giachino D, Lengauer T, Punzi L, De Marchi M: A new CARD15 mutation in Blau syndrome. Eur J Hum Genet 2005, 13:742-747.

36. Villanueva-Mendoza C, Arellanes-Garcia L, Cubas-Lorenzo V, Jimenez-Martinez MC, Flores-Suarez LF, Zenteno JC: Familial case of Blau syndrome associated with a CARD15/NOD2 mutation. Ophthalmic Genet 2010, 31:155-158.

37. Rose CD, Martin TM, Wouters CH: Blau syndrome revisited. Curr Opin Rheumatol 2011, 23:411-418.

38. Infevers Registry. [fmf.igh.cnrs.fr/infevers].

39. Okafuji I, Nishikomori R, Kanazawa N, Kambe N, Fujisawa A, Yamazaki S, Saito M, Yoshioka T, Kawai T, Sakai H, Tanizaki H, Heike T, Miyachi Y, Nakahata T: Role of the NOD2 genotype in the clinical phenotype of Blau syndrome and early-onset sarcoidosis. Arthritis Rheum 2009, 60:242-250.

40. Saulsbury FT, Wouters CH, Martin TM, Austin CR, Doyle TM, Goodwin KA, Rose CD: Incomplete penetrance of the NOD2 E383K substitution among members of a pediatric granulomatous arthritis pedigree. Arthritis Rheum 2009, 60:1804-1806.

41. Cho JH, Abraham C: Inflammatory bowel disease genetics: NOD2. Annu Rev Med 2007, 60:401-416.

42. Facco M, Cabrelle A, Teramo A, Olivieri V, Gnoato M, Teolato S, Ave E, Gattazzo C, Fadini GP, Calabrese F, Semenzato G, Agostini C: Sarcoidosis is a Th1/Th17 multisystem disorder. Thorax 2011, 66:144-150.

43. van Beelen AJ, Zelinkova Z, Taanman-Kueter EW, Muller FJ, Hommes DW Zaat SA, Kapsenberg ML, de Jong EC: Stimulation of the intracellular bacterial sensor NOD2 programs dendritic cells to promote interleukin17 production in human memory T cells. Immunity 2007, 27:660-669.

44. Zhong Y, Kinio A, Saleh M: Functions of NOD-like receptors in human diseases. Front Immunol 2013, 16:333.

45. Jun JC, Cominelli F, Abbott DW: RIP2 activity in inflammatory diseases and implications for novel therapeutics. J Leukoc Biol 2013, 94:927-932

46. Shaw MH, Reimer T, Sanchez-Valdepenas C, Warner N, Kim YG, Fresno M, Nunez G: T cell-intrinsic role of Nod2 in promoting type 1 immunity to Toxoplasma gondii. Nat Immunol 2009, 10:1267-1274.

47. Sabbah A, Chang TH, Harnack R, Frohlich V, Tominaga K, Dube PH, Xiang Y, Bose S: Activation of innate immune antiviral responses by Nod2. Nat Immunol 2009, 10:1073-1080.

48. Travassos LH, Carneiro LA, Ramjeet M, Hussey S, Kim YG, Magalhaes JG, Yuan L, Soares F, Chea E, Le Bourhis L, Boneca IG, Allaoui A, Jones NL, Nuñez G, Girardin SE, Philpott DJ: Nod1 and Nod2 direct autophagy by recruiting ATG16L1 to the plasma membrane at the site of bacterial entry. Nat Immunol 2010, 11:55-62.

49. Chamaillard M, Philpott D, Girardin SE, Zouali H, Lesage S, Chareyre F, Bui TH, Giovannini M, Zaehringer U, Penard-Lacronique V, Sansonetti PJ, Hugot JP, Thomas G: Gene-environment interaction modulated by allelic heterogeneity in inflammatory diseases. Proc Natl Acad Sci U S A 2003 100:3455-3460

50. Aksentijevich I, D Putnam C, Remmers EF, Mueller JL, Le J, Kolodner RD, Moak Z, Chuang M, Austin F, Goldbach-Mansky R, Hoffman HM, Kastner DL: The clinical continuum of cryopyrinopathies: novel CIAS1 mutations in North American patients and a new cryopyrin model. Arthritis Rheum 2007, 56:1273-1285.
51. Martin TM, Zhang Z, Kurz P, Rose CD, Chen H, Lu H, Planck SR, Davey MP, Rosenbaum JT: The NOD2 defect in Blau syndrome does not result in excess interleukin-1 activity. Arthritis Rheum 2009, 60:611-618.

52. Foley KP, Wang L, Cooper D, Magid-Slav M, Lipshutz D, Connor J, Miller M, Votta B, Gough P, Valencia X, Wouters CH, Rosé CD, Bertin J: Identification of Blau Syndrome disease signatures. Pediatr Rheumatol 2011, 9(Suppl 1):O25.

doi:10.1186/1546-0096-12-33

Cite this article as: Wouters et al:: Blau Syndrome, the prototypic auto-inflammatory granulomatous disease. Pediatric Rheumatology $201412: 33$.

\section{Submit your next manuscript to BioMed Central and take full advantage of:}

- Convenient online submission

- Thorough peer review

- No space constraints or color figure charges

- Immediate publication on acceptance

- Inclusion in PubMed, CAS, Scopus and Google Scholar

- Research which is freely available for redistribution 